\title{
ENDPOINT ESTIMATES AND WEIGHTED NORM INEQUALITIES FOR COMMUTATORS OF FRACTIONAL INTEGRALS
}

\author{
D. Cruz-Uribe, SFO And A. Fiorenza
}

Abstract

We prove that the commutator $\left[b, I_{\alpha}\right], b \in B M O, I_{\alpha}$ the fractional integral operator, satisfies the sharp, modular weak-type inequality

$\left|\left\{x \in \mathbb{R}^{n}:\left|\left[b, I_{\alpha}\right] f(x)\right|>t\right\}\right| \leq C \Psi\left(\int_{\mathbb{R}^{n}} B\left(\|b\|_{B M O} \frac{|f(x)|}{t}\right) d x\right)$,

where $B(t)=t \log (e+t)$ and $\Psi(t)=\left[t \log \left(e+t^{\alpha / n}\right)\right]^{n /(n-\alpha)}$. These commutators were first considered by Chanillo, and our result complements his. The heart of our proof consists of the pointwise inequality,

$$
M^{\#}\left(\left[b, I_{\alpha}\right] f\right)(x) \leq C\|b\|_{B M O}\left[I_{\alpha} f(x)+M_{\alpha, B} f(x)\right],
$$

where $M^{\#}$ is the sharp maximal operator, and $M_{\alpha, B}$ is a generalization of the fractional maximal operator in the scale of Orlicz spaces. Using this inequality we also prove one-weight inequalities for the commutator; to do so we prove one and two-weight norm inequalities for $M_{\alpha, B}$ which are of interest in their own right.

\section{Introduction}

Given $\alpha, 0<\alpha<n$, define the fractional integral operator $I_{\alpha}$ by

$$
I_{\alpha} f(x)=\int_{\mathbb{R}^{n}} \frac{f(y)}{|x-y|^{n-\alpha}} d y .
$$

If $b \in B M O$ we define the first order commutator $\left[b, I_{\alpha}\right]$ to be the operator

$$
\left[b, I_{\alpha}\right] f(x)=b(x) I_{\alpha} f(x)-I_{\alpha}(b f)(x)=\int_{\mathbb{R}^{n}}(b(x)-b(y)) \frac{f(y)}{|x-y|^{n-\alpha}} d y .
$$

2000 Mathematics Subject Classification. 42B20, $42 \mathrm{~B} 25$.

Key words. Fractional integrals, commutators, BMO, Orlicz spaces, maximal functions, norm inequalities.

The authors would like to thank C. Pérez for suggesting this problem, and the referee for a number of insightful comments. 
Since $b \in L^{p}(K)$ for any $p>1$ and $K$ compact, this integral converges for all $f \in C_{c}\left(\mathbb{R}^{n}\right)$.

The commutators $\left[b, I_{\alpha}\right]$ were introduced by Chanillo $[\mathbf{3}]$, who showed that for $1<p<n / \alpha, 1 / q=1 / p-\alpha / n,\left[b, I_{\alpha}\right]: L^{p}\left(\mathbb{R}^{n}\right) \rightarrow L^{q}\left(\mathbb{R}^{n}\right)$. This corresponds to the norm inequalities satisfied by $I_{\alpha}$.

The fractional integral also satisfies an endpoint inequality:

$$
\left|\left\{x \in \mathbb{R}^{n}:\left|I_{\alpha} f(x)\right|>t\right\}\right| \leq C\left(\frac{1}{t} \int_{\mathbb{R}^{n}}|f| d x\right)^{n /(n-\alpha)} .
$$

However, a straightforward computation with $f(x)=\chi_{[0,1]}$ and $b(x)=$ $\log (1+x) \chi_{(1, \infty)}$ shows that $\left[b, I_{\alpha}\right]$ is not weak $(1, n /(n-\alpha))$. (For a stronger counter-example, see Section 6 below.) Our main result is a sharp endpoint inequality for the commutator.

Theorem 1.1. Given $\alpha, 0<\alpha<n$, and a function $b \in B M O$, let $B(t)=t \log (e+t)$ and $\Psi(t)=\left[t \log \left(e+t^{\alpha / n}\right)\right]^{n /(n-\alpha)}$. Then there exists a constant $C$ such that

$$
\left|\left\{x \in \mathbb{R}^{n}:\left|\left[b, I_{\alpha}\right] f(x)\right|>t\right\}\right| \leq C \Psi\left(\int_{\mathbb{R}^{n}} B\left(\|b\|_{B M O} \frac{|f(x)|}{t}\right) d x\right) .
$$

Furthermore, this result is sharp: if (1.1) holds with $\Psi$ replaced by an increasing function $\Psi_{0}$, then there exist positive constants $\gamma$ and $K$ such that $\Psi(t / \gamma) \leq K \Psi_{0}(t), t>0$.

Remark 1.2. Since $B$ and $\Psi$ are submultiplicative, we could write the righthand side of (1.1) as

$$
C \Psi\left(B\left(\|b\|_{B M O}\right)\right) \Psi\left(\int_{\mathbb{R}^{n}} B\left(\frac{|f(x)|}{t}\right) d x\right) ;
$$

this appears more natural, but (1.1) is stronger since it is homogeneous in $b$ : we can multiply $b$ by a constant without changing the size of the constant $C$.

To prove Theorem 1.1 we first prove a pointwise inequality relating $\left[b, I_{\alpha}\right], I_{\alpha}$, and a fractional maximal operator defined using the scale of Orlicz spaces. (For precise definitions, see Section 2 below.) Given a Young function $B$ (for example, $B(t)=t \log (e+t)$ ), and $\alpha, 0 \leq \alpha<n$, define the fractional Orlicz maximal operator $M_{\alpha, B}$ by

$$
M_{\alpha, B} f(x)=\sup _{Q \ni x}|Q|^{\alpha / n}\|f\|_{B, Q},
$$


where the supremum is taken over all cubes containing $x$. When $B(t)=t$ this reduces to the classical fractional maximal operator,

$$
M_{\alpha} f(x)=\sup _{Q \ni x} \frac{|Q|^{\alpha / n}}{|Q|} \int_{Q}|f| d y .
$$

The relationship between $M_{\alpha, B}$ and $\left[b, I_{\alpha}\right]$ involves the sharp maximal function of Fefferman and Stein [11]. Recall that it is defined by

$$
M^{\#} f(x)=\sup _{Q \ni x} \frac{1}{|Q|} \int_{Q}\left|f(y)-f_{Q}\right| d y, \quad \text { where } \quad f_{Q}=\frac{1}{|Q|} \int_{Q} f(x) d x .
$$

Theorem 1.3. Let $B(t)=t \log (e+t)$. Given $\alpha, 0<\alpha<n, b \in B M O$ and a non-negative function $f$, there exists a constant $C$ such that for all $x$,

$$
M^{\#}\left(\left[b, I_{\alpha}\right] f\right)(x) \leq C\|b\|_{B M O}\left[I_{\alpha} f(x)+M_{\alpha, B} f(x)\right] .
$$

Remark 1.4. Theorem 1.3 remains true if $B(t)=t \log (e+t)$ is replaced by any "larger" Orlicz function. We will make this precise in Section 2.

Remark 1.5. Inequalities similar to (1.1) and (1.2) for singular integral operators (which formally correspond to the case $\alpha=0$ ) are true. These were first proved by Pérez $[\mathbf{2 2}]$, and our proofs are modeled on his. However, our approach to sharpness is different from his.

We can also use Theorem 1.3 to prove one-weight norm inequalities for $\left[b, I_{\alpha}\right]$. The first is a strong $(p, q)$ inequality due to Segovia and Torrea $[\mathbf{2 6}]$ which generalizes Chanillo's original result.

Theorem 1.6. Given $\alpha, 0<\alpha<n$, and $p, 1<p<n / \alpha$, fix $q$ so that $1 / q=1 / p-\alpha / n$. Let $w$ be a weight satisfying the $A_{p q}$ condition: for all cubes $Q$,

$$
\left(\frac{1}{|Q|} \int_{Q} w^{q} d x\right)^{1 / q}\left(\frac{1}{|Q|} \int_{Q} w^{-p^{\prime}} d x\right)^{1 / p^{\prime}} \leq K<\infty .
$$

Then, given any function $b \in B M O,\left[b, I_{\alpha}\right]$ satisfies the strong $(p, q)$ inequality

$$
\left(\int_{\mathbb{R}^{n}}\left|\left[b, I_{\alpha}\right] f\right|^{q} w^{q} d x\right)^{1 / q} \leq C\|b\|_{B M O}\left(\int_{\mathbb{R}^{n}}|f|^{p} w^{p} d x\right)^{1 / p} .
$$

Remark 1.7. We can also use Theorem 1.3, together with the ideas in [9] and [6], to prove two-weight norm inequalities for commutators of fractional integrals. These will be treated in a separate paper. 
The $A_{p q}$ condition governs the strong $(p, q)$ inequalities for $I_{\alpha}$; this is due to Muckenhoupt and Wheeden [18]. Given this fact, it seemed natural to conjecture that in the limiting case $p=1, q=n /(n-\alpha)$, the condition which governs the weak $(1, n /(n-\alpha))$ inequality for $I_{\alpha}$, $w^{q} \in A_{1}$, (also due to Muckenhoupt and Wheeden) would govern a weighted version of (1.1). However, this is not the case.

Example 1.8. There exists a function $w$ defined on $\mathbb{R}$ such that $w^{q} \in$ $A_{1}, q=1 /(1-\alpha)$, but there is no constant $C$ such that

$$
w^{q}\left(\left\{x \in \mathbb{R}:\left|\left[b, I_{\alpha}\right] f(x)\right|>t\right\}\right) \leq C \Psi\left(\int_{\mathbb{R}} B\left(\|b\|_{B M O} \frac{|f(x)|}{t}\right) w d x\right),
$$

where $B$ and $\Psi$ are as in Theorem 1.1, holds for all $f$.

This result is very surprising, especially since the analogous weighted inequality with $\alpha=0$ holds for singular integral operators. (See [22].) We are unsure what the correct condition on the weight $w$ should be for (1.5) to hold.

The remainder of this paper is organized as follows. In Section 2 we state some preliminary definitions and results about Orlicz spaces. In Sections 3 and 4 we state and prove an endpoint estimate and weighted norm inequalities for the Orlicz fractional maximal operator $M_{\alpha, B}$. We use these, together with Theorem 1.3, to prove Theorems 1.1 and 1.6. We actually prove results which hold for a large class of Young functions $B$, since we can do so for essentially no more work and they are of independent interest. In Section 5 we prove Theorem 1.3, in Section 6 we prove Theorem 1.1, and in Section 7 we prove Theorem 1.6, and construct Example 1.8.

Throughout this paper all notation is standard or will be defined as needed. All cubes are assumed to have their sides parallel to the coordinate axes. Given a cube $Q$ and $r>0, r Q$ will denote the cube with the same center as $Q$ and whose sides are $r$ times as long. By weights we will always mean non-negative, locally integrable functions which are positive on a set of positive measure. Given a Lebesgue measurable set $E$ and a weight $w,|E|$ will denote the Lebesgue measure of $E$ and $w(E)=\int_{E} w d x$. Given $1<p<\infty, p^{\prime}=p /(p-1)$ will denote the conjugate exponent of $p$. $C$ and $c$ will denote positive constants whose value may change at each appearance.

Finally, we assume that the reader is familiar with the definition and basic properties of the Hardy-Littlewood maximal operator $M$, its dyadic variant $M^{d}$, and the Muckenhoupt $A_{p}$ weights, $1 \leq p \leq \infty$. We refer the reader to $[\mathbf{1 0}]$ or $[\mathbf{1 3}]$ for further information. 


\section{Background on Orlicz spaces}

In the following we are going to use some notions of Orlicz space theory. Here we summarize some basic facts; we refer the reader to [16], [17], or $[\mathbf{2 4}]$ for further details.

A function $B:[0, \infty) \rightarrow[0, \infty)$ is a Young function if it is continuous, convex and strictly increasing, and if $B(0)=0, B(t) \rightarrow \infty$ as $t \rightarrow \infty$.

If $A, B$ are Young functions, we write $A(t) \approx B(t)$ if there are constants $t_{0}, c_{1}, c_{2}>0$ such that $c_{1} A(t) \leq B(t) \leq c_{2} A(t)$ for $t \geq t_{0}$. Also, we say that $B$ dominates $A$, and denote this by $A \preceq B$, if there exists $c>0$ such that for all $t>0, A(t) \leq B(c t)$. If this is true for all $t \geq t_{0}>0$, we say that $A \preceq B$ near infinity.

A Young function $B$ is said to be doubling if there exists a positive constant $C$ such that $B(2 t) \leq C B(t)$ for all $t>0 ; B$ is called submultiplicative if $B(s t) \leq C B(s) B(t)$ for all $s, t>0$. Clearly $B(t)=t^{r}$, $r \geq 1$, is submultiplicative. A straightforward computation shows that $B(t)=t^{a}[\log (e+t)]^{b}, a \geq 1, b>0$, is also submultiplicative. (For simplicity, hereafter we will omit the brackets and write simply $t^{a} \log (e+t)^{b}$.)

Given a non-empty open set $E$ in $\mathbb{R}^{n}$ and a Young function $B$, the Orlicz space $L_{B}(E)$ is the Banach space of Lebesgue measurable functions $f$ such that $B(|f| / \lambda)$ is (Lebesgue) integrable on $E$ for some $\lambda>0$. It is equipped with the Luxemburg norm

$$
\|f\|_{L_{B}(E)}=\inf \left\{\lambda>0: \int_{E} B\left(\frac{|f|}{\lambda}\right) d x \leq 1\right\} .
$$

When $E$ has finite measure (e.g. it is a cube) we often want to normalize by replacing the measure $d x$ by $d x /|E|$. In particular, given a cube $Q$, we define the mean Luxemburg norm of $f$ on $Q$ by

$$
\|f\|_{B, Q}=\inf \left\{\lambda>0: \frac{1}{|Q|} \int_{Q} B\left(\frac{|f|}{\lambda}\right) d x \leq 1\right\} .
$$

When $B(t)=t^{r}, 1 \leq r<\infty$,

$$
\|f\|_{B, Q}=\left(\frac{1}{|Q|} \int_{Q}|f|^{r} d x\right)^{1 / r},
$$

so the Luxemburg norm coincides with the (normalized) $L^{r}$ norm.

If $A \preceq B$ near infinity then there exists a constant $C$, depending on $A$ and $B$, such that for all cubes $Q$ and functions $f,\|f\|_{A, Q} \leq C\|f\|_{B, Q}$. This follows from the standard embedding theorem which shows that $L_{B}(Q) \subset L_{A}(Q)$. However, we stress that because this is the mean Luxemburg norm, the constant $C$ is independent of $Q$. 
It follows from this that if $A \preceq B$ near infinity, then $M_{\alpha, A} f(x) \leq$ $C M_{\alpha, B} f(x)$. In particular, in Theorem 1.3 we can take $B$ to be any Young function such that $t \log (e+t) \preceq B$ near infinity. (This makes precise Remark 1.4.)

Given a Young function $B$, the complementary Young function $\bar{B}$ is defined by

$$
\bar{B}(t)=\sup _{s>0}\{s t-B(s)\}, \quad t>0 .
$$

$B$ and $\bar{B}$ satisfy the following inequality: $t \leq B^{-1}(t) \bar{B}^{-1}(t) \leq 2 t$.

We will need a generalization of Hölder's inequality to Orlicz spaces due to O'Neil [19]. (Also see [17] or [24].)

Lemma 2.1. Given a Young function $B$, then for all functions $f$ and $g$ and all cubes $Q$,

$$
\frac{1}{|Q|} \int_{Q}|f g| d x \leq 2\|f\|_{B, Q}\|g\|_{\bar{B}, Q}
$$

More generally, if $A, B$ and $C$ are Young functions such that for all $t>0$,

$$
B^{-1}(t) C^{-1}(t) \leq A^{-1}(t),
$$

then

$$
\|f g\|_{A, Q} \leq 2\|f\|_{B, Q}\|g\|_{C, Q} .
$$

If we set $g \equiv 1$ in (2.3), it immediately follows that for all Young functions $B, \alpha, 0 \leq \alpha<n$, and $x \in \mathbb{R}^{n}$,

$$
M_{\alpha} f(x) \leq C M_{\alpha, B} f(x) .
$$

\section{Endpoint inequality for $M_{\alpha, B}$}

In this section we prove a modular endpoint inequality for the Orlicz fractional maximal operator which we need for the proof of Theorem 1.1. To state it we need the following definition.

Definition 3.1. Given a Young function $B$, define the function $h_{B}$ by

$$
h_{B}(s)=\sup _{t>0} \frac{B(s t)}{B(t)}, \quad 0 \leq s<\infty .
$$

Remark 3.2. The function $h_{B}$ could be infinite if $s>1$, but if $B$ is doubling then it is finite for all $0<s<\infty$. (See [17, Theorem 11.7].) If $B$ is submultiplicative then $h_{B} \approx B$. More generally, given any $B$, for all $s, t \geq 0, B(s t) \leq h_{B}(s) B(t)$. 
Theorem 3.3. Given $\alpha, 0 \leq \alpha<n$, let $B$ be a Young function such that $B(t) / t^{n / \alpha}$ is decreasing for all $t>0$. Then there exists a constant depending only on $B$ such that for all $t>0, M_{\alpha, B}$ satisfies the modular weak-type inequality

$$
\Phi\left(\left|\left\{x \in \mathbb{R}^{n}: M_{\alpha, B} f(x)>t\right\}\right|\right) \leq C \int_{\mathbb{R}^{n}} B\left(\frac{f(x)}{t}\right) d x,
$$

for all non-negative $f \in L_{B}\left(\mathbb{R}^{n}\right)$, where $\Phi$ is any function such that

$$
\Phi(s) \leq C_{1} \Phi_{1}(s)= \begin{cases}0 & \text { if } s=0 \\ \frac{s}{h_{B}\left(s^{\alpha / n}\right)} & \text { if } s>0 .\end{cases}
$$

Before proving Theorem 3.3 we make a number of observations about its statement.

Remark 3.4. When $\alpha=0$ we interpret the growth condition to mean that $B$ can be any Young function.

Remark 3.5. The function $\Phi_{1}$ is well-defined: by Lemma 3.12 below, it follows from the fact that $B(t) / t^{n / \alpha}$ is decreasing that $0<h_{B}\left(s^{\alpha / n}\right)<\infty$ for all $s>0$. Also note that if $B$ is submultiplicative, $B \approx h_{B}$, so $\Phi_{1}(s) \approx s / B\left(s^{\alpha / n}\right)$.

Remark 3.6. Suppose $\Phi$ is continuous and invertible. If we let $\Psi=\Phi^{-1}$, and if $\Psi$ is doubling, then inequality (3.1) can be written as

$$
\left|\left\{x \in \mathbb{R}^{n}: M_{\alpha, B} f(x)>t\right\}\right| \leq C \Psi\left(\int_{\mathbb{R}^{n}} B\left(\frac{f(x)}{t}\right) d x\right) .
$$

By Lemma 3.12, for any $B$ satisfying the assumptions of Theorem 3.3 there exist functions $\Phi$, invertible, satisfying $\Phi(s) \leq C \Phi_{1}(s)$. In the proof of Theorem 1.1 we use (3.2) with $B(t)=t \log (e+t)$.

Remark 3.7. We can weaken the growth condition on $B$ as follows: if $I(B)<n / \alpha$, where $I(B)$ denotes the upper Boyd index of $B$, then there exists a Young function $B_{1}$ such that $B_{1} \approx B$ and $B_{1}(t) / t^{n / \alpha}$ is decreasing. See [12, Theorem 1.1] for further details.

Remark 3.8. When $B(t)=t, \Phi(t)=t^{1-\alpha / n}$ (or $\Psi(t)=t^{n /(n-\alpha)}$ in (3.2)), and Theorem 3.3 reduces to the weak $(1, n /(n-\alpha))$ inequality for the classical fractional maximal operator (cf. [10, p. 89]). When $\alpha=0$, $\Phi(t)=\Psi(t)=t$, and (3.1) becomes the modular endpoint inequality for $M_{0, B}$ due to Pérez $[\mathbf{2 1}]$. 
Remark 3.9. In the proof of Theorem 1.3 we need Theorem 3.3 for the case $B(t)=t \log (e+t)$. Since $B$ is submultiplicative, we can take

$$
\Phi(t)=\frac{t^{1-\alpha / n}}{\log \left(e+t^{\alpha / n}\right)},
$$

or equivalently,

$$
\Psi(t)=\left[t \log \left(e+t^{\alpha / n}\right)\right]^{n /(n-\alpha)} .
$$

We could replace $t^{\alpha / n}$ by $t$ in the definition of $\Phi$ and $\Psi$; we chose not to since with the given definitions we recapture the case $\alpha=0$.

Remark 3.10. In the limiting case $B(t)=t^{n / \alpha}$, Theorem 3.3 is trivial: for all $x \in \mathbb{R}^{n}$,

$$
M_{\alpha, B} f(x)=\sup _{Q \ni x}|Q|^{\alpha / n}\left(\frac{1}{|Q|} \int_{Q}|f|^{n / \alpha} d x\right)^{\alpha / n}=\left(\int_{\mathbb{R}^{n}}|f|^{n / \alpha} d x\right)^{\alpha / n},
$$

and therefore $M_{\alpha, B} f$ is constant. Then (3.1) is trivially true with $\Phi$ defined by $\Phi(0)=0, \Phi(s)=1, s>0$.

The proof of Theorem 3.3 requires four lemmas.

Lemma 3.11. If $B$ is a Young function then $h_{B}$ is nonnegative, submultiplicative, increasing in $[0, \infty)$, strictly increasing in $[0,1]$ and $h_{B}(1)=1$.

For the (easy) proof see [6, Lemma 3.1] or [17, p. 84].

Lemma 3.12. Given $\alpha, 0 \leq \alpha<n$, let $B$ be a Young function such that $B(t) / t^{n / \alpha}$ is decreasing for all $t>0$. Then the function $\Phi_{1}$ in Theorem 3.3 is increasing, and $\Phi_{1}(s) / s$ is decreasing. Moreover, there exists $\Phi$ such that $\Phi(s) \leq C_{1} \Phi_{1}(s)$ and $\Phi$ is invertible.

Proof of Lemma 3.12: If $\alpha=0$, the assertion is trivial. If $0<\alpha<n$, for $0<s<\sigma$ and $t>0, s^{\alpha / n} t<\sigma^{\alpha / n} t$. Since the function $B(t) / t^{n / \alpha}$ is decreasing,

therefore,

$$
\frac{B\left(\sigma^{\alpha / n} t\right)}{\sigma t^{n / \alpha}} \leq \frac{B\left(s^{\alpha / n} t\right)}{s t^{n / \alpha}}
$$

$$
\frac{B\left(\sigma^{\alpha / n} t\right)}{\sigma B(t)} \leq \frac{B\left(s^{\alpha / n} t\right)}{s B(t)} .
$$

If we take the supremum over all $t$, then

$$
\frac{h_{B}\left(\sigma^{\alpha / n}\right)}{\sigma} \leq \frac{h_{B}\left(s^{\alpha / n}\right)}{s} .
$$

It follows immediately that $\Phi_{1}(s) \leq \Phi_{1}(\sigma)$. Furthermore, by Lemma 3.11, $\Phi_{1}(s) / s$ is decreasing. 
Finally, if $C(s)$ is any continuous and strictly increasing function such that $C(0)=1, C(s) \rightarrow 2$ as $s \rightarrow+\infty$, then trivially the function $\Phi$ defined by $\Phi(s)=C(s) \Phi_{1}(s)$ is invertible and satisfies $\Phi(s) \leq 2 \Phi_{1}(s)$ since $\Phi_{1}(s)>0$ if $s>0$.

Lemma 3.13. If $\Phi(t) / t$ is decreasing, then for any positive sequence $\left\{x_{j}\right\}$,

$$
\Phi\left(\sum_{j} x_{j}\right) \leq \sum_{j} \Phi\left(x_{j}\right) .
$$

For a proof, see [14, p. 83, n. 103].

Lemma 3.14. Given a non-negative, locally integrable function $f$ and $\alpha, 0 \leq \alpha<n$, suppose that for some Young function $B$, cube $Q$ and $t>0$,

$$
|Q|^{\alpha / n}\|f\|_{B, Q}>t .
$$

Then there exists a dyadic cube $P$ such that $Q \subset 3 P$ and a positive constant $\beta_{\alpha, n}$, depending only on $\alpha$ and $n$, such that

$$
|P|^{\alpha / n}\|f\|_{B, P}>\beta_{\alpha, n} t .
$$

When $B(t)=t$ this result is proved in [5], and when $\alpha=0$ this is implicit in [21, Lemma 4.1]. Either proof readily adapts and we omit the details.

Proof of Theorem 3.3: Fix a non-negative function $f$ in $L_{B}\left(\mathbb{R}^{n}\right)$. Fix $t>0$ and define

$$
E_{t}=\left\{x \in \mathbb{R}^{n}: M_{\alpha, B} f(x)>t\right\} .
$$

If $t$ is such that the set $E_{t}$ is empty, we have nothing to prove. Otherwise, for each $x \in E_{t}$ there exists a cube $Q_{x}$ containing $x$ such that

$$
\left|Q_{x}\right|^{\alpha / n}\|f\|_{B, Q_{x}}>t .
$$

By Lemma 3.14, there is a constant $\beta$ such that for each $x$ there exists a dyadic cube $P_{x}$ with $Q_{x} \subset 3 P_{x}$ and

$$
\left|P_{x}\right|^{\alpha / n}\|f\|_{B, P_{x}}>\beta t .
$$

Since $f \in L_{B}\left(\mathbb{R}^{n}\right)$, we can replace the collection $\left\{P_{x}\right\}$ with a maximal disjoint subcollection $\left\{P_{j}\right\}$. Each $P_{j}$ satisfies (3.3), and by our choice of the $Q_{x}$ 's, $E_{t} \subset \bigcup_{j} 3 P_{j}$. 
By Lemmas 3.12 and 3.13,

$$
\Phi_{1}\left(\left|E_{t}\right|\right) \leq \Phi_{1}\left(\sum_{j}\left|3 P_{j}\right|\right) \leq \sum_{j} \Phi_{1}\left(\left|3 P_{j}\right|\right) .
$$

On the other hand, inequality (3.3) implies that for each $j$,

$$
\left\|(\beta t)^{-1}\left|P_{j}\right|^{\alpha / n} f\right\|_{B, P_{j}}>1,
$$

and so by the definition of the Luxemburg norm (2.1) and by Definition 3.1,

$$
\begin{aligned}
1 & <\frac{1}{\left|P_{j}\right|} \int_{P_{j}} B\left(\frac{\left|P_{j}\right|^{\alpha / n} f(x)}{\beta t}\right) d x \\
& \leq \frac{1}{\left|P_{j}\right|} \int_{P_{j}} h_{B}\left(\left|P_{j}\right|^{\alpha / n} \beta^{-1}\right) B\left(\frac{f(x)}{t}\right) d x \\
& \leq \frac{h_{B}\left(\beta^{-1}\right) h_{B}\left(\left|P_{j}\right|^{\alpha / n}\right)}{\left|P_{j}\right|} \int_{P_{j}} B\left(\frac{f(x)}{t}\right) d x \\
& \leq 3^{n} \frac{h_{B}\left(\beta^{-1}\right) h_{B}\left(\left|3 P_{j}\right|^{\alpha / n}\right)}{\left|3 P_{j}\right|} \int_{P_{j}} B\left(\frac{f(x)}{t}\right) d x \\
& =3^{n} \frac{h_{B}\left(\beta^{-1}\right)}{\Phi_{1}\left(\left|3 P_{j}\right|\right)} \int_{P_{j}} B\left(\frac{f(x)}{t}\right) d x .
\end{aligned}
$$

Hence, since the $P_{j}$ 's are disjoint,

$$
\begin{aligned}
\sum_{j} \Phi_{1}\left(\left|3 P_{j}\right|\right) & \leq 3^{n} h_{B}\left(\beta^{-1}\right) \sum_{j} \int_{P_{j}} B\left(\frac{f(x)}{t}\right) d x \\
& \leq 3^{n} h_{B}\left(\beta^{-1}\right) \int_{\mathbb{R}^{n}} B\left(\frac{f(x)}{t}\right) d x
\end{aligned}
$$

Inequality (3.1) now follows at once.

Remark 3.15. If $B$ is a submultiplicative Young function, then inequality (3.1) is sharp in the sense that $\Phi_{1}$ cannot be replaced by an essentially larger function, at least for $x$ large. To show this we construct a simple example. Fix $n=1,0<\alpha<1$, and $x$ such that $x^{\alpha}>B^{-1}(1)$. Now fix $N=x / B\left(x^{\alpha}\right)<x$; the reason for this choice will be clear below. If we let $f=\chi_{[0, N]}$, then for all $y \in[0, x]$,

$$
M_{\alpha, B} f(y) \geq x^{\alpha}\|f\|_{B,[0, x]}=\frac{x^{\alpha}}{B^{-1}(x / N)}=1 .
$$


In particular,

$$
[0, x] \subset\left\{y \in \mathbb{R}: M_{\alpha, B} f(y)>1 / 2\right\} .
$$

Hence, if (3.1) holds for some function $\Phi$, then, since $B$ is submultiplicative we must have that for all $x$ sufficiently large,

$$
\begin{aligned}
\Phi(x) & \leq \Phi\left(\left|\left\{y \in \mathbb{R}: M_{\alpha, B} f(y)>1 / 2\right\}\right|\right) \\
& \leq C \int_{\mathbb{R}} B(2 f(x)) d x \\
& =C B(2) N \\
& \leq C \Phi_{1}(x) ;
\end{aligned}
$$

the last inequality follows from Remark 3.5.

We conjecture that for a submultiplicative Young function $B, \Phi_{1}$ is the best possible function for all $x$, and not just $x$ large, but we cannot prove this. However, we can prove this for a large class of such functions. Let $B$ be a submultiplicative Young function such that

$$
\liminf _{x \rightarrow 0} \limsup _{z \rightarrow \infty} \frac{B(z x)}{B(z) B(x)}>c>0 .
$$

Intuitively, (3.4) implies that $B$ is essentially multiplicative for $z$ large and $x$ small. A large number of Young functions have this property: for example, a straightforward computation using L'Hôpital's rule shows that for $a \geq 1, b>0, B(t)=t^{a} \log (e+t)^{b}$ satisfies (3.4).

However, not every submultiplicative Young function satisfies (3.4). For instance, given $1<\alpha<\beta$, the function

$$
B(t)= \begin{cases}t^{\alpha} & 0<x<1 \\ t^{\beta} & x \geq 1\end{cases}
$$

is submultiplicative, but (3.4) does not hold:

$$
\liminf _{x \rightarrow 0} \limsup _{z \rightarrow \infty} \frac{B(z x)}{B(z) B(x)}=\liminf _{x \rightarrow 0} \limsup _{z \rightarrow \infty} \frac{(z x)^{\beta}}{z^{\beta} x^{\alpha}}=0 .
$$


Given a submultiplicative Young function $B$ which satisfies (3.4), we modify the above argument as follows. Since $\alpha>0$, condition (3.4) implies that there exist $x_{0}, z_{0}>0, z_{0}$ depending on $x$, such that if $0<x<x_{0}$ and $z>z_{0}$, then

$$
\frac{B\left(z x^{\alpha}\right)}{B(z) B\left(x^{\alpha}\right)}>c .
$$

Fix $x<x_{0}$ and choose $N>0$ such that $0<N<x$ and $2 B^{-1}(x / N) x^{-\alpha}>$ $z_{0}$. Again let $f=\chi_{[0, N]}$. If we fix $t>0$ such that

$$
2 t=\frac{x^{\alpha}}{B^{-1}(x / N)},
$$

then we have that for $y \in[0, x], M_{\alpha, B} f(x)>t$. We now argue as before to get that

$$
\Phi(x) \leq C N B\left(\frac{2 B^{-1}(x / N)}{x^{\alpha}}\right) .
$$

If we let $z=2 B^{-1}(x / N) x^{-\alpha}$ in (3.5), then since $B$ is submultiplicative we get

$$
\begin{aligned}
& \leq C N \frac{B\left(2 B^{-1}(x / N)\right)}{B\left(x^{\alpha}\right)} \\
& \leq C \frac{x}{B\left(x^{\alpha}\right)} \\
& \leq C \Phi_{1}(x) .
\end{aligned}
$$

Remark 3.16. If a Young function $B$ is not submultiplicative we suspect that $\Phi_{1}$ is still the best possible, but we have no evidence to support this.

\section{Weighted norm inequalities for $M_{\alpha, B}$}

In this section we prove weighted norm inequalities for $M_{\alpha, B}$ which we will need to prove Theorem 1.6. We establish a more general, two-weight result which is interesting in its own right.

Definition 4.1. Given $p, 1<p<\infty$, we say that a Young function $B$ satisfies the $B_{p}$ condition if there exists a constant $c>0$ such that

$$
\int_{c}^{\infty} \frac{B(t)}{t^{p+1}} d t<\infty .
$$


Theorem 4.2. Given $p, q, 1<p \leq q<\infty$, and $\alpha, 0 \leq \alpha<n$, let $A, B$ and $C$ be Young functions such that $A^{-1}(t) C^{-1}(t) \leq B^{-1}(t)$, and such that $C$ is doubling and satisfies the $B_{p}$ condition. If $(u, v)$ is a pair of weights such that for every cube $Q$,

$$
|Q|^{\alpha / n+1 / q-1 / p}\left(\frac{1}{|Q|} \int_{Q} u^{q} d x\right)^{1 / q}\left\|v^{-1}\right\|_{A, Q} \leq K<\infty,
$$

then for all $f \in L^{p}\left(v^{p}\right)$,

$$
\left(\int_{\mathbb{R}^{n}}\left(M_{\alpha, B} f\right)^{q} u^{q} d x\right)^{1 / q} \leq C\left(\int_{\mathbb{R}^{n}}|f|^{p} v^{p} d x\right)^{1 / p} .
$$

Remark 4.3. The case $\alpha=0$ and $p=q$ was first proved by Cruz-Uribe and Pérez $[8]$. Our proof is adapted from theirs.

We will need the following corollary in the proof of Theorem 1.6.

Corollary 4.4. Given $\alpha, 0<\alpha<n$, and $p, 1<p<n / \alpha$, fix $q$ so that $1 / q=1 / p-\alpha / n$. Let $w$ be a weight satisfying the $A_{p q}$ condition (1.3), and let $B(t)=t \log (e+t)$. Then for all $f \in L^{p}\left(w^{p}\right)$,

$$
\left(\int_{\mathbb{R}^{n}}\left(M_{\alpha, B} f\right)^{q} w^{q} d x\right)^{1 / q} \leq C\left(\int_{\mathbb{R}^{n}}|f|^{p} w^{p} d x\right)^{1 / p} .
$$

Remark 4.5. In the special case when $u=v=1$, then $M_{\alpha, B}: L^{p} \rightarrow L^{q}$ if $B \in B_{p}$. We give a short proof of this fact. We first claim that if $B \in B_{p}$ then $B \preceq t^{p}$ near infinity. To see this, note that by Definition 4.1, there exists $t_{0}>0$ such that if $t \geq t_{0}$ then

$$
\int_{t}^{2 t} \frac{B(s)}{s^{p+1}} d s \leq 1
$$

Since $B$ is increasing, this implies that

$$
1 \geq \frac{B(t)}{(2 t)^{p}} \int_{t}^{2 t} \frac{d s}{s}=\frac{B(t)}{(2 t)^{p}} \ln (2) .
$$

Hence, $B \preceq t^{p}$ near infinity.

Now, given any cube $Q$,

$$
\begin{aligned}
|Q|^{\alpha / n}\|f\|_{B, Q} & =|Q|^{\alpha / n}\|f\|_{B, Q}^{\alpha p / n}\|f\|_{B, Q}^{1-\alpha p / n} \\
& \leq C|Q|^{\alpha / n}\left(\frac{1}{|Q|} \int_{Q}|f|^{p} d y\right)^{\alpha / n}\|f\|_{B, Q}^{1-\alpha p / n} \\
& \leq C\|f\|_{p}^{1-p / q}\|f\|_{B, Q}^{p / q} .
\end{aligned}
$$


Hence, for all $x$,

$$
M_{\alpha, B} f(x) \leq C\|f\|_{p}^{1-p / q} M_{0, B} f(x)^{p / q} .
$$

Pérez $[\mathbf{2 1}]$ showed that if $B \in B_{p}$ then $M_{0, B}$ is bounded on $L^{p}$. Therefore,

$$
\begin{aligned}
\int_{\mathbb{R}^{n}}\left(M_{\alpha, B} f\right)^{q} d x & \leq C\|f\|_{p}^{q-p} \int_{\mathbb{R}^{n}}\left(M_{0, B} f\right)^{p} d x \\
& \leq C\|f\|_{p}^{q-p} \int_{\mathbb{R}^{n}}|f|^{p} d x \\
& =C\|f\|_{p}^{q},
\end{aligned}
$$

which is what we wanted to show.

The proof of Theorem 4.2 requires the following lemma.

Lemma 4.6. Fix $\alpha, 0 \leq \alpha<n$. Given a Young function B, suppose $f$ is a non-negative function such that $|Q|^{\alpha / n}\|f\|_{B, Q}$ tends to zero as $|Q|$ tends to infinity. Given $a>2^{n+1+\alpha / n}$, for each $k \in \mathbb{Z}$ there exists a disjoint collection of maximal dyadic cubes $\left\{Q_{j}^{k}\right\}$ such that for each $j$,

$$
a^{k}<\left|Q_{j}^{k}\right|^{\alpha / n}\|f\|_{B, Q_{j}^{k}} \leq 2^{n+\alpha / n} a^{k},
$$

and there exists $\beta>1$, depending only on $n$ and $\alpha$, such that

$$
\left\{x \in \mathbb{R}^{n}: M_{\alpha, B} f(x)>\beta a^{k}\right\} \subset \bigcup_{j} 3 Q_{j}^{k} .
$$

Further, let $D_{k}=\bigcup_{j} Q_{j}^{k}$ and $E_{j}^{k}=Q_{j}^{k} \backslash\left(Q_{j}^{k} \cap D_{k+1}\right)$. Then the $E_{j}^{k}$ 's are pairwise disjoint for all $j$ and $k$ and there exists a constant $\gamma>1$, depending only on a and $\gamma$, such that $\left|Q_{j}^{k}\right| \leq \gamma\left|E_{j}^{k}\right|$.

Remark 4.7. To have $f$ satisfy the hypotheses of Lemma 4.6, it suffices to assume that $f$ is bounded and has compact support.

The case $\alpha=0$ of Lemma 4.6 was proved in [8]; the same proof, with the obvious changes, works for $0<\alpha<n$, and we omit the details.

Proof of Theorem 4.2: Fix a function $f$; without loss of generality we may assume that $f$ is non-negative, bounded, and has compact support. Fix $a>2^{n+1+\alpha / n}$ and for $k \in \mathbb{Z}$ let

$$
\Omega_{k}=\left\{x \in \mathbb{R}^{n}: M_{\alpha, B} f(x)>\beta a^{k}\right\} .
$$


For each $k$ form the collection of dyadic cubes $\left\{Q_{j}^{k}\right\}$ in Lemma 4.6. Then we can estimate as follows:

$$
\begin{aligned}
\int_{\mathbb{R}^{n}}\left(M_{\alpha, B} f\right)^{q} u^{q} d x & =\sum_{k} \int_{\Omega_{k} \backslash \Omega_{k+1}}\left(M_{\alpha, B} f\right)^{q} u^{q} d x \\
& \leq C \sum_{k} a^{k q} u^{q}\left(\Omega_{k}\right) \\
& \leq C \sum_{k, j} a^{k q} u^{q}\left(3 Q_{j}^{k}\right) \\
& \leq C \sum_{k, j} u^{q}\left(3 Q_{j}^{k}\right)\left|Q_{j}^{k}\right|^{q \alpha / n}\|f\|_{B, Q_{j}^{k}}^{q} \\
& =C \sum_{k, j} u^{q}\left(3 Q_{j}^{k}\right)\left|Q_{j}^{k}\right|^{q \alpha / n}\left\|f v \cdot v^{-1}\right\|_{B, Q_{j}^{k}}^{q} .
\end{aligned}
$$

By Lemmas 2.1 and 4.6, and by (4.1),

$$
\begin{aligned}
& \leq C \sum_{k, j}\left|Q_{j}^{k}\right|^{q \alpha / n} u^{q}\left(3 Q_{j}^{k}\right)\left\|v^{-1}\right\|_{A, Q_{j}^{k}}^{q}\|f v\|_{C, Q_{j}^{k}}^{q} \\
& \leq C \sum_{k, j}\left|3 Q_{j}^{k}\right|^{q \alpha / n}\left(\frac{1}{\left|3 Q_{j}^{k}\right|} \int_{3 Q_{j}^{k}} u^{q} d x\right) \\
& \quad \times\left\|v^{-1}\right\|_{A, 3 Q_{j}^{k}}^{q}\|f v\|_{C, Q_{j}^{k}}^{q}\left|E_{j}^{k}\right| \\
& \leq C \sum_{k, j}\|f v\|_{C, Q_{j}^{k}}^{q}\left|E_{j}^{k}\right|^{q / p} .
\end{aligned}
$$

Since $q / p \geq 1$ and the $E_{j}^{k}$ 's are pairwise disjoint,

$$
\begin{aligned}
& \leq C\left(\sum_{k, j}\|f v\|_{C, Q_{j}^{k}}^{p}\left|E_{j}^{k}\right|\right)^{q / p} \\
& \leq C\left(\sum_{k, j} \int_{E_{j}^{k}} M_{0, C}(f v)^{p} d x\right)^{q / p} \\
& \leq C\left(\int_{\mathbb{R}^{n}} M_{0, C}(f v)^{p} d x\right)^{q / p} .
\end{aligned}
$$


As we noted above, since $C$ satisfies the $B_{p}$ condition, $M_{0, C}$ is bounded on $L^{p}$ (see $[\mathbf{2 1}]$ ); hence

$$
\leq C\left(\int_{\mathbb{R}^{n}}|f|^{p} v^{p} d x\right)^{q / p} .
$$

This completes our proof.

Proof of Corollary 4.4: Fix $\alpha, p$ and $q$ as in the hypotheses. It will suffice to show that if $w \in A_{p q}$ then the pair $(w, w)$ satisfies the hypotheses of Theorem 4.2.

Let $r=1+p^{\prime} / q$; then we can re-write the $A_{p q}$ condition as

$$
\left(\frac{1}{|Q|} \int_{Q} w^{-p^{\prime}} d x\right)\left(\frac{1}{|Q|} \int_{Q}\left(w^{-p^{\prime}}\right)^{1-r^{\prime}} d x\right)^{r-1} \leq K<\infty .
$$

Hence $w^{-p^{\prime}}$ is in $A_{r}$, and so satisfies the reverse Hölder inequality with exponent $s>1$. Let $A(t)=t^{s p^{\prime}}$; then $A^{-1}(t)=t^{1 / s p^{\prime}}$. Therefore, if we let

$$
C^{-1}(t)=\frac{t^{1 /\left(s p^{\prime}\right)^{\prime}}}{\log (e+t)},
$$

we have that

$$
A^{-1}(t) C^{-1}(t)=\frac{t}{\log (t)} \approx B^{-1}(t) .
$$

Furthermore,

$$
C(t) \approx(t \log (e+t))^{\left(s p^{\prime}\right)^{\prime}}
$$

since $s p^{\prime}>p^{\prime},\left(s p^{\prime}\right)^{\prime}<p$, and so $C$ satisfies the $B_{p}$ condition.

Inequality (4.1) now follows at once:

$$
\begin{aligned}
|Q|^{\alpha / n+1 / q-1 / p} & \left(\frac{1}{|Q|} \int_{Q} w^{q} d x\right)^{1 / q}\left\|v^{-1}\right\|_{A, Q} \\
& =\left(\frac{1}{|Q|} \int_{Q} w^{-p^{\prime}} d x\right)^{1 / q}\left(\frac{1}{|Q|} \int_{Q} w^{-p^{\prime} s} d x\right)^{1 / s p^{\prime}} \\
& \leq C\left(\frac{1}{|Q|} \int_{Q} w^{-p^{\prime}} d x\right)^{q}\left(\frac{1}{|Q|} \int_{Q} w^{-p^{\prime}} d x\right)^{1 / p^{\prime}} \\
& \leq K
\end{aligned}
$$




\section{Proof of Theorem 1.3}

Our proof requires several facts about functions in $B M O$ and about the fractional integral operator. We gather these in two lemmas.

Lemma 5.1. The following are true:

(1) A function $b$ is in BMO if for each cube $Q$ there exists a constant $c_{Q}$ such that

$$
\sup _{Q} \frac{1}{|Q|} \int_{Q}\left|b(x)-c_{Q}\right| d x<\infty .
$$

Further, this supremum is comparable to $\|b\|_{B M O}$.

(2) For each $p, 1<p<\infty$, there exists a constant $C_{p}$ such that

$$
\sup _{Q}\left(\frac{1}{|Q|} \int_{Q}\left|b(x)-b_{Q}\right|^{p} d x\right)^{1 / p} \leq C_{p}\|b\|_{B M O} .
$$

(3) If $b \in B M O$ then there exists a constant $C$ such that for every cube $Q$,

$$
\frac{1}{|Q|} \int_{Q} \exp \left(\frac{\left|b(x)-b_{Q}\right|}{C\|b\|_{B M O}}\right) d x<\infty .
$$

(4) If $b \in B M O$ then for any cube $Q$ and $k \geq 0$,

$$
\left|b_{2^{k+1} Q}-b_{Q}\right| \leq 2(k+1)\|b\|_{B M O} .
$$

For a proof of (1)-(3), see [10, Chapter 6]. For a proof of $(4)$, see [27, p. 206].

Lemma 5.2. Given $\alpha, 0<\alpha<n$, and a non-negative function $f$, the following are true:

(1) There exists a constant $C$ such that for any cube $Q$,

$$
\int_{Q} I_{\alpha} f d x \leq C|Q|^{\alpha / n} \int_{\mathbb{R}^{n}} f d x
$$

(2) $I_{\alpha} f \in A_{1}$, that is, there exists a constant $C$ such that $M\left(I_{\alpha} f\right)(x) \leq$ $C I_{\alpha} f(x)$ for almost every $x$. Hence, it satisfies the reverse Hölder inequality for some exponent $s>1$.

(3) $I_{\alpha}$ is weak $(1, n /(n-\alpha))$ : for all $\lambda>0$,

$$
\left|\left\{x \in \mathbb{R}^{n}:\left|I_{\alpha}(x)\right|>\lambda\right\}\right| \leq C\left(\frac{1}{\lambda} \int_{\mathbb{R}^{n}}|f(x)| d x\right)^{n /(n-\alpha)} .
$$


Proof: Inequality (5.1) follows easily from Fubini's theorem:

$$
\begin{aligned}
\int_{Q} I_{\alpha} f d x & =\int_{Q} \int_{\mathbb{R}^{n}} \frac{f(y) d y}{|x-y|^{n-\alpha}} d x \\
& =\int_{\mathbb{R}^{n}} f(y)\left[\int_{Q}|x-y|^{\alpha-n} d x\right] d y \\
& \leq C|Q|^{\alpha / n} \int_{\mathbb{R}^{n}} f(x) d x
\end{aligned}
$$

To see that $I_{\alpha} f \in A_{1}$, it suffices to note that $|x|^{\alpha-n} \in A_{1}$, and the convolution of a non-negative function with an $A_{1}$ weight is again in $A_{1}$. (Cf. [25].) For the weak $(1, n /(n-\alpha))$ inequality, see $[\mathbf{1 0}$, p. 89].

Proof of Theorem 1.3: By homogeneity, it will suffice to prove (1.2) for $x=0$. Further, by Lemma 5.2(2), and Lemma 5.1(1), it will suffice to show that, given a cube $Q$ centered at the origin, there exists a constant $c_{Q}$ such that

$$
\frac{1}{|Q|} \int_{Q}\left|\left[b, I_{\alpha}\right] f(y)-c_{Q}\right| d y \leq C\|b\|_{B M O}\left[M\left(I_{\alpha} f\right)(0)+M_{\alpha, B} f(0)\right]
$$

Decompose $f$ as $f_{1}+f_{2}$, where $f_{1}=f \chi_{Q^{*}}$, and $Q^{*}$ is the cube centered at the origin whose sides are $2 \sqrt{n}$ times larger. Let $c_{Q}=\left(I_{\alpha}\left(\left(b-b_{Q^{*}}\right) f_{2}\right)\right)_{Q}$. Then, since $\left[b, I_{\alpha}\right] f=\left[b-b_{Q^{*}}, I_{\alpha}\right] f$,

$$
\begin{aligned}
& \frac{1}{|Q|} \int_{Q}\left|\left[b, I_{\alpha}\right] f(y)-c_{Q}\right| d y \\
& \leq \frac{1}{|Q|} \int_{Q}\left|\left(b(y)-b_{Q^{*}}\right) I_{\alpha} f(y)\right| d y \\
&+\frac{1}{|Q|} \int_{Q}\left|I_{\alpha}\left(\left(b-b_{Q^{*}}\right) f_{1}\right)(y)\right| d y \\
&+\frac{1}{|Q|} \int_{Q}\left|I_{\alpha}\left(\left(b-b_{Q^{*}}\right) f_{2}\right)(y)-\left(I_{\alpha}\left(\left(b-b_{Q^{*}}\right) f_{2}\right)\right)_{Q}\right| d y \\
&= I_{1}+I_{2}+I_{3} .
\end{aligned}
$$

We estimate each integral in turn. For $I_{1}$, by Lemma $5.2(2), I_{\alpha} f$ satisfies the reverse Hölder inequality with exponent $s>1$. Therefore, 
if we apply Hölder's inequality with exponent $s$, by Lemma 5.1(2),

$$
\begin{aligned}
I_{1} & \leq\left(\frac{1}{|Q|} \int_{Q}\left|\left(b(y)-b_{Q^{*}}\right)\right|^{s^{\prime}} d y\right)^{1 / s^{\prime}}\left(\frac{1}{|Q|} \int_{Q} I_{\alpha} f(y)^{s} d y\right)^{1 / s} \\
& \leq C\|b\|_{B M O}\left(\frac{1}{|Q|} \int_{Q} I_{\alpha} f(y) d y\right) \\
& \leq C\|b\|_{B M O} M\left(I_{\alpha} f\right)(0) .
\end{aligned}
$$

To estimate the second integral, note that by Lemma 5.1(3),

$$
\frac{1}{\left|Q^{*}\right|} \int_{Q^{*}} \exp \left(\frac{\left|b(y)-b_{Q^{*}}\right|}{C\|b\|_{B M O}}\right) d y<\infty
$$

Since $B(t)=t \log (e+t), \bar{B}(t) \preceq e^{t}-1$; therefore, by $(2.1)$,

$$
\left\|b-b_{Q^{*}}\right\|_{\bar{B}, Q^{*}} \leq C\|b\|_{B M O} .
$$

Hence, by Lemma 5.2(1), and by the generalized Hölder inequality (2.3),

$$
\begin{aligned}
I_{2} & \leq \frac{C|Q|^{\alpha / n}}{|Q|} \int_{\mathbb{R}^{n}}\left|b(y)-b_{Q^{*}}\right| f_{1}(y) d y \\
& =\frac{C\left|Q^{*}\right|^{\alpha / n}}{\left|Q^{*}\right|} \int_{Q^{*}}\left|b(y)-b_{Q^{*}}\right| f(y) d y \\
& \leq C\left|Q^{*}\right|^{\alpha / n}\left\|b-b_{Q^{*}}\right\|_{\bar{B}, Q^{*}}\|f\|_{B, Q^{*}} \\
& \leq C\|b\|_{B M O} M_{\alpha, B} f(0) .
\end{aligned}
$$

Finally, we estimate the third integral. By the mean-value theorem, if $|x|>2|h|$ then there exists $\theta, 0 \leq \theta \leq 1$, such that

$$
\left|\frac{1}{|x|^{n-\alpha}}-\frac{1}{|x+h|^{n-\alpha}}\right| \leq C \frac{|h|}{|x+\theta h|^{n-\alpha+1}} \leq C \frac{|h|}{|x|^{n-\alpha+1}} .
$$


If $x, y \in Q$ and $z \in \mathbb{R}^{n} \backslash 2^{k} Q^{*}, k \geq 0$, then $|x-z|>2^{k+1}|y-x|$.

Therefore,

$$
\begin{aligned}
I_{3} \leq & \frac{1}{|Q|} \int_{Q} \frac{1}{|Q|} \int_{Q} \int_{\mathbb{R}^{n} \backslash Q^{*}}\left|\left(b(z)-b_{Q^{*}}\right) f(z)\right| \frac{1}{|y-z|^{n-\alpha}}-\frac{1}{|x-z|^{n-\alpha}} \mid d z d x d y \\
\leq & \frac{1}{|Q|^{2}} \int_{Q} \int_{Q} \sum_{k=0}^{\infty} \int_{2^{k+1} Q^{*} \backslash 2^{k} Q^{*}}\left|\left(b(z)-b_{Q^{*}}\right) f(z)\right| \frac{|y-x|}{|x-z|^{n-\alpha+1}} d z d x d y \\
\leq & \frac{C}{|Q|^{2}} \int_{Q} \int_{Q} \sum_{k=0}^{\infty} \frac{2^{-k}}{\left|2^{k+1} Q^{*}\right|^{1-\alpha / n}} \int_{2^{k+1} Q^{*}}\left|\left(b(z)-b_{Q^{*}}\right) f(z)\right| d z d x d y \\
\leq & C \sum_{k=0}^{\infty} \frac{2^{-k}}{\left|2^{k+1} Q^{*}\right| 1-\alpha / n} \int_{2^{k+1} Q^{*}}\left|\left(b(z)-b_{2^{k+1} Q^{*}}\right) f(z)\right| d z \\
& +C \sum_{k=0}^{\infty} \frac{2^{-k}}{\left|2^{k+1} Q^{*}\right| 1-\alpha / n} \int_{2^{k+1} Q^{*}}\left|\left(b_{2^{k+1} Q^{*}}-b_{Q^{*}}\right) f(z)\right| d z \\
\leq & \sum_{k=0}^{\infty} 2^{-k}\left|2^{k+1} Q^{*}\right|^{\alpha / n}\left\|b-b_{2^{k+1} Q^{*}}\right\|_{\bar{B}, 2^{k+1} Q^{*}}\|f\|_{B, 2^{k+1} Q^{*}} \\
& +C\|b\|_{B M O} \sum_{k=0}^{\infty} \frac{(k+1) 2^{-k}}{\left|2^{k+1} Q^{*}\right| 1-\alpha / n} \int_{2^{k+1} Q^{*}}|f(z)| d z \\
\leq & C\|b\|_{B M O} M_{\alpha, B} f(0) . \\
& \quad C M_{\alpha, B} f(0)+\|_{B M O} M_{\alpha} f(0)
\end{aligned}
$$

The fifth inequality follows from Lemma 5.1(4), and the last inequality follows from (2.5). This completes the proof.

\section{Proof of Theorem 1.1}

In our proof we need a variant of the good- $\lambda$ inequality of Fefferman and Stein $[\mathbf{1 1}]$ relating the dyadic maximal operator and the sharp maximal operator. (Also see [10, p. 121].) This exact result is given by Pérez [22]; the proof is a straightforward modification of the proof of the standard result and so is omitted. 
Lemma 6.1. Let $\varphi$ be a positive function on $(0, \infty)$ such that $\varphi(2 t) \leq$ $\varphi(t)$ for all $t>0$. Then there exists a positive constant $C$ such that

$$
\sup _{\lambda>0} \varphi(\lambda)\left|\left\{y \in \mathbb{R}^{n}: M^{d} f(y)>\lambda\right\}\right| \leq C \sup _{\lambda>0} \varphi(\lambda)\left|\left\{y \in \mathbb{R}^{n}: M^{\#} f(y)>\lambda\right\}\right|
$$

for all functions such that the lefthand side is finite.

Proof of Theorem 1.1: First, fix a function in BMO. If $\|b\|_{B M O}=0$ then $b$ is constant and the result is trivial. We may therefore assume that $\|b\|_{B M O} \neq 0$.

Now fix a function $f$. Since we can decompose an arbitrary function into the sum of its positive and negative parts, without loss of generality we may assume that $f$ is non-negative. By homogeneity, it will suffice to prove (1.1) when $t=1$, that is,

(6.1) $\left|\left\{x \in \mathbb{R}^{n}:\left|\left[b, I_{\alpha}\right] f(x)\right|>1\right\}\right| \leq C \Psi\left(\int_{\mathbb{R}^{n}} B\left(\|b\|_{B M O}|f(x)|\right) d x\right)$.

But in this case,

$$
\begin{aligned}
\mid\left\{x \in \mathbb{R}^{n}\right. & \left.:\left|\left[b, I_{\alpha}\right] f(x)\right|>1\right\} \mid \\
& \leq \Psi(B(1)) \sup _{t>0} \frac{1}{\Psi(B(1 / t))}\left|\left\{x \in \mathbb{R}^{n}:\left|\left[b, I_{\alpha}\right] f(x)\right|>t\right\}\right| \\
& \leq \Psi(B(1)) \sup _{t>0} \frac{1}{\Psi(B(1 / t))}\left|\left\{x \in \mathbb{R}^{n}: M^{d}\left(\left[b, I_{\alpha}\right] f\right)(x)>t\right\}\right| .
\end{aligned}
$$

Let $\varphi(t)=1 / \Psi(B(1 / t))$; then a straightforward but somewhat tedious calculation shows that

$$
\lim _{t \rightarrow 0} \frac{\varphi(2 t)}{\varphi(t)}=\lim _{t \rightarrow \infty} \frac{\varphi(2 t)}{\varphi(t)}=2^{n /(n-\alpha)}
$$


and so $\varphi(2 t) \leq C \varphi(t)$ for all $t>0$. Therefore, by Lemma 6.1 and Theorem 1.3,

$$
\begin{aligned}
\mid\left\{x \in \mathbb{R}^{n}\right. & \left.:\left|\left[b, I_{\alpha}\right] f(x)\right|>1\right\} \mid \\
\leq & C \sup _{t>0} \frac{1}{\Psi(B(1 / t))}\left|\left\{x \in \mathbb{R}^{n}: M^{\#}\left(\left[b, I_{\alpha}\right] f\right)(x)>t\right\}\right| \\
\leq & C \sup _{t>0} \frac{1}{\Psi(B(1 / t))}\left|\left\{x \in \mathbb{R}^{n}: I_{\alpha} f(x)+M_{\alpha, B} f(x)>\frac{t}{C \|_{B M O}}\right\}\right| \\
\leq & C \sup _{t>0} \frac{1}{\Psi(B(1 / t))}\left|\left\{x \in \mathbb{R}^{n}: I_{\alpha} f(x)>\frac{t}{C\|b\|_{B M O}}\right\}\right| \\
& \quad+C \sup _{t>0} \frac{1}{\Psi(B(1 / t))}\left|\left\{x \in \mathbb{R}^{n}: M_{\alpha, B} f(x)>\frac{t}{C\|b\|_{B M O}}\right\}\right| .
\end{aligned}
$$

By (5.2) and (3.2), and since $\Psi$ and $B$ are submultiplicative,

$$
\begin{aligned}
\leq & C \sup _{t>0} \frac{1}{\Psi(B(1 / t))}\left(\frac{\|b\|_{B M O}}{t} \int_{\mathbb{R}^{n}}|f(x)| d x\right)^{n /(n-\alpha)} \\
& +C \sup _{t>0} \frac{1}{\Psi(B(1 / t))} \Psi\left(\int_{\mathbb{R}^{n}} B\left(\|b\|_{B M O} \frac{|f(x)|}{t}\right) d x\right) \\
\leq & C \sup _{t>0} \frac{1}{\Psi(B(1 / t))} \cdot \frac{1}{t^{n /(n-\alpha)}}\left(\int_{\mathbb{R}^{n}}\|b\|_{B M O}|f(x)| d x\right)^{n /(n-\alpha)} \\
& +C \sup _{t>0} \frac{1}{\Psi(B(1 / t))} \cdot \Psi(B(1 / t)) \Psi\left(\int_{\mathbb{R}^{n}} B\left(\|b\|_{B M O}|f(x)|\right) d x\right) \\
= & J_{1}+J_{2} .
\end{aligned}
$$

Note that

$$
\sup _{t>0} \frac{1}{\Psi(B(1 / t))} \cdot \frac{1}{t^{n /(n-\alpha)}} \leq C,
$$

since

$$
\frac{1}{\Psi(B(1 / t))} \cdot \frac{1}{t^{n /(n-\alpha)}}=\frac{1}{\left[\log (e+1 / t) \log \left(e+[(1 / t) \log (e+1 / t)]^{\alpha / n}\right)\right]^{\frac{n}{n-\alpha}}}
$$

is continuous and has finite limits as $t \rightarrow 0, t \rightarrow \infty$ ( 0 and 1 respectively). 
Furthermore, since $t \leq B(t)$ and $t^{n /(n-\alpha)} \leq \Psi(t)$, we have that

$$
\left(\int_{\mathbb{R}^{n}}\|b\|_{B M O}|f(x)| d x\right)^{n /(n-\alpha)} \leq \Psi\left(\int_{\mathbb{R}^{n}} B\left(\|b\|_{B M O}|f(x)|\right) d x\right) .
$$

From (6.2) and (6.3) we get that

$$
J_{1}+J_{2} \leq C \Psi\left(\int_{\mathbb{R}^{n}} B\left(\|b\|_{B M O}|f(x)|\right) d x\right),
$$

which proves (6.1).

We will now show that (1.1) is sharp, in the sense that if we can replace $\Psi$ by $\Psi_{0}$, then for all $t>0, \Psi(t / \gamma) \leq K \Psi_{0}(t)$. To do so, we will adapt the argument in Remark 3.15, which explored sharpness in Theorem 3.3. Let $n=1$ and $0<\alpha<1$, fix $x>0$ and let $N$ be such that $0<N<x$; the exact value of $N$ will be chosen below. Let $f=\chi_{[0, N]}$.

First, we show that there is a constant $K$, depending only on $\alpha$ such that for all $y>N$,

$$
M_{\alpha, B} f(y) \leq \frac{K y^{\alpha}}{B^{-1}(y / N)} .
$$

(As we showed in Remark 3.15, the opposite inequality holds with constant 1.) To see this, note that since $f$ is a non-increasing function on $[0, \infty)$,

$$
M_{\alpha, B} f(y)=\sup _{z \geq y} z^{\alpha}\|f\|_{B,[0, z]}=\sup _{z \geq y} \frac{z^{\alpha}}{B^{-1}(z / N)} .
$$

Therefore, it will suffice to show that there exists $K>0$, such that if $z \geq y$

$$
\frac{z^{\alpha}}{B^{-1}(z / N)} \leq \frac{K y^{\alpha}}{B^{-1}(y / N)} .
$$

Let $H(t)=t^{\alpha} / B^{-1}(t / N)$; since for all $t>0, B^{-1}(t) \approx t / \log (e+t)$, we have that

$$
\frac{H(z)}{H(y)} \leq C \frac{(z / N)^{\alpha-1} \log (e+z / N)}{(y / N)^{\alpha-1} \log (e+y / N)} .
$$

The function $\log (e+t) / t^{1-\alpha}$ is either decreasing or has a unique local maximum on $[1, \infty)$; it follows, therefore, that the righthand term is dominated by a constant which depends only on $\alpha$. This establishes (6.4).

Now let $b(y)=\log (e+y / N) \chi_{(N, \infty)}(y)$. Since the $B M O$ norm is dilation invariant, the $B M O$ norm of $b$ does not depend on $N$. For all $z$, 
$I_{\alpha}(b f)(z)=0$, so for all $y$ such that $N<y<x$,

$$
\begin{aligned}
\left|\left[b, I_{\alpha}\right] f(y)\right| & =b(y) I_{\alpha} f(y) \\
& =\log (e+y / N) \int_{0}^{N} \frac{d z}{|z-y|^{1-\alpha}} \\
& \geq \frac{N \log (e+y / N)}{y^{1-\alpha}} \\
& \geq \frac{c y^{\alpha}}{B^{-1}(y / N)}
\end{aligned}
$$

by inequality (6.4),

$$
\begin{aligned}
& \geq c M_{\alpha, B} f(y) \\
& >\frac{c x^{\alpha}}{B^{-1}(x / N)} .
\end{aligned}
$$

The constant $c$ depends only on $\alpha$.

We now consider two cases, depending on whether $x$ is large or small. Suppose first that $x$ is such that $c x^{\alpha}>B^{-1}(2)$, and let $N=$ $x / B\left(c x^{\alpha}\right)<x / 2$. Then the above inequality shows that for all $y \in$ $[x / 2, x],\left[b, I_{\alpha}\right] f(y)>1$. Hence, if inequality (1.1) holds for some increasing function $\Psi_{0}$ then we have that

$$
\begin{aligned}
x / 2 & \leq\left|\left\{y \in \mathbb{R}:\left|\left[b, I_{\alpha}\right] f(y)\right|>1\right\}\right| \\
& \leq C \Psi_{0}\left(\int_{\mathbb{R}} B\left(\|b\|_{B M O} f(y)\right) d y\right) \\
& \leq C \Psi_{0}\left(N B\left(\|b\|_{B M O}\right)\right) .
\end{aligned}
$$

On the other hand, there is a constant $\gamma$, depending only on $\alpha$, such that

$$
B\left(\|b\|_{B M O}\right) N=\frac{B\left(\|b\|_{B M O}\right) x}{B\left(c x^{\alpha}\right)} \leq \gamma \Phi\left(c^{1 / \alpha} x\right),
$$

where $\Phi=\Psi^{-1}$. Therefore, if set the righthand side equal to $t$ and solve for $x$, we get that

$$
c^{1 / \alpha} x=\Psi(t / \gamma) .
$$

If we combine this with the inequality above we get that for all $t$ sufficiently large, $\Psi(t / \gamma) \leq K \Psi_{0}(t)$.

We will now show that the same inequality holds for all $t$ sufficiently small. Fix $x>0$ small and let $0<N<x / 2$; the exact value of $N$ will be 
fixed below. Then, by the above argument we have that if $N<y<x$,

$$
\left[b, I_{\alpha}\right] f(y) \geq \frac{c x^{\alpha}}{B^{-1}(x / N)} .
$$

Therefore, if we fix $u$ such that

$$
2 u=\frac{c x^{\alpha}}{B^{-1}(x / N)},
$$

then arguing as above and as in Remark 3.15, we have that

$$
\begin{aligned}
x / 2 & \leq C \Psi_{0}\left(\int_{\mathbb{R}} B\left(\|b\|_{B M O} \frac{f(y)}{u}\right) d y\right) \\
& =C \Psi_{0}\left(N B\left(\|b\|_{B M O} \frac{2 B^{-1}(x / N)}{c x^{\alpha}}\right)\right) .
\end{aligned}
$$

Again arguing as in Remark 3.15 we can choose $N$ sufficiently small such that

$$
\begin{aligned}
& \leq C \Psi_{0}\left(C B\left(\|b\|_{B M O}\right) x^{1-\alpha}\right) \\
& \leq C \Psi_{0}\left(C B\left(\|b\|_{B M O}\right) \Phi(x)\right) .
\end{aligned}
$$

We can now argue as we did above to get that for all $t$ sufficiently small, $\Psi(t / \gamma) \leq K \Psi_{0}(t)$. This completes our proof.

\section{Proof of Theorem 1.6 and Construction of Example 1.8}

Our proof of Theorem 1.6 requires two facts which we give as a lemma.

Lemma 7.1. The following are true:

(1) If $w \in A_{p}$ for some $p>1$, then for all $q>0$, there exists a constant $C_{q}$ such that

$$
\int_{\mathbb{R}^{n}}\left(M^{d} f\right)^{q} w d x \leq C_{q} \int_{\mathbb{R}^{n}}\left(M^{\#} f\right)^{q} w d x .
$$

(2) Given $\alpha, 0<\alpha<n, p, 1<p<n / \alpha$, let $1 / q=1 / p-\alpha / n$. Then if $w \in A_{p q}$,

$$
\left(\int_{\mathbb{R}^{n}}\left|I_{\alpha} f\right|^{q} w^{q} d x\right)^{1 / q} \leq C\left(\int_{\mathbb{R}^{n}}|f|^{p} w^{p} d x\right)^{1 / p} .
$$

The first inequality is due to Journé [15]; also see [10, p. 144]. The second is due to Muckenhoupt and Wheeden [18]. 
Proof of Theorem 1.6: Fix a function $f$; as in the proof of Theorem 1.1, we may assume without loss of generality that $f$ is non-negative. Now, arguing as we did in Section 4 in the proof of Corollary 4.4, if $w \in A_{p q}$ then $w^{q} \in A_{r}, r=1+q / p^{\prime}$. Therefore, by Lemma 7.1 and Corollary 4.4,

$$
\begin{aligned}
\left(\int_{\mathbb{R}^{n}}\left|\left[b, I_{\alpha}\right] f\right|^{q} w^{q} d x\right)^{1 / q} \leq & \left(\int_{\mathbb{R}^{n}} M^{d}\left(\left[b, I_{\alpha}\right] f\right)^{q} w^{q} d x\right)^{1 / q} \\
\leq & C\left(\int_{\mathbb{R}^{n}} M^{\#}\left(\left[b, I_{\alpha}\right] f\right)^{q} w^{q} d x\right)^{1 / q} \\
\leq & C\|b\|_{B M O}\left(\int_{\mathbb{R}^{n}}\left(I_{\alpha} f\right)^{q} w^{q} d x\right)^{1 / q} \\
& +C\|b\|_{B M O}\left(\int_{\mathbb{R}^{n}}\left(M_{\alpha, B} f\right)^{q} w^{q} d x\right)^{1 / q} \\
\leq & C\|b\|_{B M O}\left(\int_{\mathbb{R}^{n}} f^{p} w^{p} d x\right)^{1 / p} .
\end{aligned}
$$

Construction of Example 1.8: For clarity, we let $\alpha=1 / 2$; the following example can be readily adapted to any $\alpha, 0<\alpha<1$. We will show that there exists a function $w$ such that $w^{q} \in A_{1}$ and such that

$$
w^{q}\left(\left\{x \in \mathbb{R}: M_{\alpha, B} f(x)>t\right\}\right) \leq C \Psi\left(\int_{\mathbb{R}} B\left(\frac{|f(x)|}{t}\right) w(x) d x\right)
$$

$B(t)=t \log (e+t), \Psi(t)=t^{2} \log \left(e+t^{1 / 2}\right)^{2}$, fails to hold with a uniform constant for all $f$. For then an argument almost identical to that at the end of the proof of Theorem 1.1 shows that (1.5) cannot hold with a uniform constant.

The desired function $w$ is $w(x)=|x|^{-1 / 4}$. Then $w^{q}(x)=w(x)^{2}=$ $|x|^{-1 / 2} \in A_{1}$. (Cf. $\left[\mathbf{1 0}\right.$, p. 141].) For $N \geq 2$, let $Q=\left[N^{4}-N, N^{4}\right]$. Fix $x_{0}>N$ such that

$$
N=\frac{x_{0}}{B\left(x_{0}^{\alpha}\right)}=\frac{x_{0}^{1 / 2}}{\log \left(e+x_{0}^{1 / 2}\right)} .
$$

(Since $\Theta(t)=t^{1 / 2} / \log \left(e+t^{1 / 2}\right)$ is increasing, we can always find such an $x_{0}$.) Let $\bar{Q}=\left[N^{4}-N, N^{4}-N+x_{0}\right]$. Now define $f=\chi_{Q}$; since $f$ is the translation of $\chi_{[0, N]}$, the function which appears in Remark 3.15, 
the argument given there shows that if $y \in \bar{Q}, M_{\alpha, B} f(y) \geq 1$. Hence,

$$
\bar{Q} \subset\left\{x \in \mathbb{R}: M_{\alpha, B} f(x)>1 / 2\right\} .
$$

Let $t=1 / 2$ in (7.1); we estimate each side in turn. The righthand side is a constant independent of $N \geq 2$ :

$$
\begin{aligned}
\Psi\left(\int_{\mathbb{R}} B(2|f(x)|) w(x) d x\right) & =\Psi\left(\int_{Q} B(2) x^{-1 / 4} d x\right) \\
& \leq \Psi\left(\frac{B(2) N}{\left(N^{4}-N\right)^{1 / 4}}\right) \\
& \leq C .
\end{aligned}
$$

The lefthand side of (7.1) is unbounded. Since $x_{0}=\Theta^{-1}(N) \approx$ $N^{2} \log \left(e+N^{1 / 2}\right)^{2}$, there exists $c>0$ such that

$$
\begin{aligned}
w^{q}\left(\left\{x \in \mathbb{R}: M_{\alpha, B} f(x)>1 / 2\right\}\right) & \geq w^{q}(\bar{Q}) \\
& \geq \int_{N^{4}}^{N^{4}+c N^{2} \log \left(e+N^{1 / 2}\right)^{2}} x^{-1 / 2} d x \\
& \geq \frac{c N^{2} \log \left(e+N^{1 / 2}\right)^{2}}{\left(N^{4}+c N^{2} \log \left(e+N^{1 / 2}\right)^{2}\right)^{1 / 2}} \\
& \geq c \log \left(e+N^{1 / 2}\right)^{2} .
\end{aligned}
$$

Therefore, as $N$ tends to infinity, (7.1) cannot hold with a uniform constant and we are done.

\section{References}

[1] D. R. Adams, Weighted nonlinear potential theory, Trans. Amer. Math. Soc. 297(1) (1986), 73-94.

[2] C. Bennett and R. Sharpley, "Interpolation of operators", Pure and Applied Mathematics 129, Academic Press, Inc., Boston, MA, 1988.

[3] S. Chanillo, A note on commutators, Indiana Univ. Math. J. 31(1) (1982), 7-16.

[4] R. R. Coifman and R. Rochberg, Another characterization of BMO, Proc. Amer. Math. Soc. 79(2) (1980), 249-254.

[5] D. Cruz-Uribe, SFO, New proofs of two-weight norm inequalities for the maximal operator, Georgian Math. J. 7(1) (2000), 33-42. 
[6] D. Cruz-Uribe, SFO And A. Fiorenza, The $A_{\infty}$ property for Young functions and weighted norm inequalities, Houston J. Math. 28(1) (2002), 169-182.

[7] D. Cruz-Uribe, SFO And C. J. Neugebauer, The structure of the reverse Hölder classes, Trans. Amer. Math. Soc. 347(8) (1995), 2941-2960.

[8] D. Cruz-Uribe, SFO And C. PÉrez, Sharp two-weight, weaktype norm inequalities for singular integral operators, Math. Res. Lett. 6(3-4) (1999), 417-427.

[9] D. Cruz-Uribe, SFO and C. PÉrez, Two-weight, weak-type norm inequalities for fractional integrals, Calderón-Zygmund operators and commutators, Indiana Univ. Math. J. 49(2) (2000), 697-721.

[10] J. Duonndikomtxea, "Fourier analysis", Graduate Studies in Mathematics 29, American Mathematical Society, Providence, RI, 2001.

[11] C. Fefferman and E. M. Stein, Some maximal inequalities, Amer. J. Math. 93 (1971), 107-115.

[12] A. Fiorenza And M. Krbec, Indices of Orlicz spaces and some applications, Comment. Math. Univ. Carolin. 38(3) (1997), 433-451.

[13] J. García-Cuerva And J. L. Rubio de Francia, "Weighted norm inequalities and related topics", North-Holland Mathematics Studies 116, Notas de Matemática 104, North-Holland Publishing Co., Amsterdam, 1985.

[14] G. H. Hardy, J. E. Littlewood and G. Pólya, "Inequalities", 2d ed., Cambridge University Press, Cambridge, 1952.

[15] J.-L. JouRné, "Calderón-Zygmund operators, pseudodifferential operators and the Cauchy integral of Calderón", Lecture Notes in Mathematics 994, Springer-Verlag, Berlin, 1983.

[16] M. A. Krasnosel'skiI And Ya. B. RutickIĬ, “Convex functions and Orlicz spaces", P. Noordhoff Ltd., Groningen, 1961.

[17] L. Maligranda, Orlicz spaces and interpolation, Seminars in Mathematics 5, IMECC, Universidad Estadual de Campinas, Campinas, Brazil (1989).

[18] B. Muckenhoupt And R. Wheeden, Weighted norm inequalities for fractional integrals, Trans. Amer. Math. Soc. 192 (1974), $261-274$.

[19] R. O'NeIL, Fractional integration in Orlicz spaces. I, Trans. Amer. Math. Soc. 115 (1965), 300-328. 
[20] C. PÉrez, Two weighted inequalities for potential and fractional type maximal operators, Indiana Univ. Math. J. 43(2) (1994), 663-683.

[21] C. PÉREZ, On sufficient conditions for the boundedness of the Hardy-Littlewood maximal operator between weighted $L^{p}$-spaces with different weights, Proc. London Math. Soc. (3) 71(1) (1995), $135-157$.

[22] C. PÉREz, Endpoint estimates for commutators of singular integral operators, J. Funct. Anal. 128(1) (1995), 163-185.

[23] C. Pérez and G. Pradolini, Sharp weighted endpoint estimates for commutators of singular integrals, Michigan Math. J. 49(1) (2001), 23-37.

[24] M. M. Rao and Z. D. Ren, "Theory of Orlicz spaces", Monographs and Textbooks in Pure and Applied Mathematics 146, Marcel Dekker, Inc., New York, 1991.

[25] E. T. SAwYeR, A two weight weak type inequality for fractional integrals, Trans. Amer. Math. Soc. 281(1) (1984), 339-345.

[26] C. Segovia and J. L. Torrea, Weighted inequalities for commutators of fractional and singular integrals, Conference on Mathematical Analysis in honor of the memory of José Luis Rubio de Francia (El Escorial, 1989), Publ. Mat. 35(1) (1991), 209-235.

[27] A. Torchinsky, "Real-variable methods in harmonic analysis", Pure and Applied Mathematics 123, Academic Press, Inc., Orlando, FL, 1986.

D. Cruz-Uribe, SFO:

Department of Mathematics

Trinity College

Hartford, CT 06106-3100

U.S.A.

E-mail address: david.cruzuribe@mail.trincoll.edu

A. Fiorenza:

Dipartimento di Costruzioni e Metodi Matematici in Architettura

Universitá di Napoli

Via Monteoliveto, 3

I-80134 Napoli

Italy

E-mail address: fiorenza@unina.it

Primera versió rebuda el 28 de gener de 2002, darrera versió rebuda el 31 de maig de 2002. 